\title{
Mass loss of massive stars near the Eddington luminosity by core neutrino emission shortly before their explosion
}

\author{
Takashi J. Moriya ${ }^{1,2}$ \\ 1 Argelander Institute for Astronomy, University of Bonn, Auf dem Hügel 71, 53121 Bonn, Germany \\ e-mail: moriyatk@astro.uni-bonn.de \\ 2 Research Center for the Early Universe, Graduate School of Science, University of Tokyo, Hongo 7-3-1, Bunkyo, 113-033 Tokyo, \\ Japan
}

Received 6 November 2013 / Accepted 11 March 2014

\begin{abstract}
We present a novel mechanism for enhancing the mass-loss rates of massive stars shortly before their explosion. The neutrino luminosities of the stellar core of massive stars increase as they get closer to the time of the core collapse. As emitted neutrinos escape freely from the core, the core mass is significantly reduced when the neutrino luminosity is high. If a star is near the Eddington luminosity when the neutrino luminosity is high, the star can exceed the Eddington luminosity because of the core neutrino mass loss. We suggest that the stellar surface mass-loss rates due to the core neutrino emission can be higher than $10^{-4} M_{\odot} \mathrm{yr}^{-1}$ from $\sim 1$ year before the core collapse. The mass-loss rates can exceed $10^{-2} M_{\odot} \mathrm{yr}^{-1} \sim 10$ days before the core collapse. This mass-loss mechanism may be able to explain the enhanced mass loss observed in some supernova progenitors shortly before their explosion. Even if the star is not close enough to the Eddington luminosity to enhance the mass loss, the star can still expand because of the reduced gravitational force. This mechanism can be activated in Wolf-Rayet stars, and it can create the hydrogen-poor, as well as hydrogen-rich, dense circumstellar media observed in some supernovae.
\end{abstract}

Key words. stars: massive - stars: mass-loss - supernovae: general - stars: evolution

\section{Introduction}

Stellar mass loss is one of the most influential phenomena that determine the fate of a massive star. For instance, the spectral type of an exploding star, a supernova $(\mathrm{SN})$, depends on the amount of mass loss during the evolution. There is growing evidence that some stars experience very high mass-loss rates shortly before the explosion. SN 2006jc is, for the first time, found to have got bright two years before its explosion as a Type Ibn SN (Pastorello et al. 2007). A similar Type Ibn SN iPTF13beo is found to have two light curve peaks that indicate that the mass-loss rate of the Wolf-Rayet (WR) progenitor is $\sim 2 \times 10^{-3} M_{\odot} \mathrm{yr}^{-1}$ in a year before the explosion (Gorbikov et al. 2013). Type IIn SNe are more commonly discovered SNe with dense circumstellar media (CSM) resulting from the high mass-loss rates of their progenitors shortly before their explosions. They are typically higher than $\sim 10^{-3} M_{\odot} \mathrm{yr}^{-1}$ (e.g., Taddia et al. 2013; Kiewe et al. 2012; Moriya et al. 2013).

Having such high mass-loss rates immediately before the explosions of these SN progenitors is not expected from the standard stellar evolution theory, and its mechanisms are still not known well. Some of them are likely to be related to the progenitors' very high luminosities and their proximity to the Eddington luminosity. The progenitor of Type IIn SN $2005 \mathrm{gl}$ is found to have an absolute brightness of $M_{V} \simeq-10 \mathrm{mag}$ and it belongs to the most luminous class of stars. The progenitor of Type IIn SN 2009ip provided us with more remarkable information about the progenitors' activities (e.g., Margutti et al. 2014; Smith et al. 2014; Mauerhan et al. 2013; Prieto et al. 2013; but see also Fraser et al. 2013a; Pastorello et al. 2013). The luminosity of the progenitor was again close to $M_{V}=-10 \mathrm{mag}$, and it is presumed to be near the Eddington luminosity. The progenitor started to show several outbursts from a few years before its explosion. The final burst in August 2012 started about 50 days before the major luminosity increase started in September 2012, which may have been caused by the SN explosion. A dense CSM could have been formed during these eruptive events. Type IIn SNe $2010 \mathrm{mc}$ and $2011 \mathrm{ht}$ also experienced a similar pre-SN outburst (Ofek et al. 2013; Fraser et al. 2013b). A Type Ic superluminous SN 2006oz had a precursor from about ten days before its major luminosity increase (Leloudas et al. 2012). This precursor may also be triggered by the progenitor activities shortly before the explosion (but see also Moriya \& Maeda 2012 for another interpretation).

In addition to the unexpected mass-loss enhancement shortly before the core collapse, some stars may have larger radii than those expected from the stellar evolution theory at the time of the explosion. The early emission of Type Ib SN 2008D is said to be difficult to explain by standard WR stars (e.g., Dessart et al. 2011). Bersten et al. (2013) show that the radius of the WR progenitor of SN $2008 \mathrm{D}$ should be $\sim 10 R_{\odot}$ to explain the early emission by the adiabatic cooling of the SN ejecta, and they conclude that ${ }^{56} \mathrm{Ni}$ may have been pumped out to the outermost layers of the WR progenitor (see also, e.g., Couch et al. 2011; Balberg \& Loeb 2011 for other interpretations). Some fast evolving Type Ic SNe are said to originate in extended WR stars whose radii are $\sim 10 R_{\odot}$ (Kleiser \& Kasen 2013 , but see also Tauris et al. 2013). The progenitors of ultra-long gammaray bursts are unlikely to be WR stars with their typical radii because of their long durations (e.g., Levan et al. 2014). Those 
observations may infer that some WR stars explode with much larger radii than those predicted by the current stellar evolution theory.

One of the characteristics of massive stars shortly before the explosion is their huge neutrino luminosities. Neutrinos are constantly emitted from the stellar core throughout the evolution because of the nuclear reactions. However, after the onset of the carbon burning, the thermal neutrino emission becomes significant because of the high temperature required for igniting heavy elements. The expected neutrino luminosity is so high that dying stars will be detected by the modern neutrino detectors from a few weeks before the core collapse if they are close enough to the Earth (Odrzywolek et al. 2011, 2004). The high neutrino luminosity from the core, together with the energy release by nuclear burning and the composition gradients, results in the active convective motion that is said to be related to the high mass-loss rates of SN progenitors (e.g., Quataert \& Shiode 2012; Shiode \& Quataert 2014). Here, in this paper, we suggest another possible effect of the huge neutrino luminosity, which may result in the extreme mass loss at the stellar surface. The mass loss of the core due to the neutrino emission leads to the sudden decrease of the gravitational force. The effect of the mass loss due to high neutrino luminosities after the core collapse of a star has been discussed in Lovegrove \& Woosley (2013) and Nadezhin (1980). In this paper, we suggest that the effect of the neutrino mass loss at the stellar core can appear at the stellar surface in massive stars near the Eddington luminosity immediately before their core collapse. In short, the gravitational potential can be significantly reduced because of the neutrino emission shortly before the core collapse, and the stellar luminosity can exceed the Eddington luminosity. Thus, the weakened gravitational force results in the enhanced mass loss. It may also result in the expansion of massive stars shortly before their explosion.

\section{Neutrino mass loss from the stellar core}

At the very late stage of the stellar evolution, especially after the onset of the core carbon burning, the neutrino luminosity from the stellar core starts to be dominated by thermal neutrinos. The emerged neutrino freely escapes from the core. The mass-loss rate $\dot{M}_{\mathrm{c}}$ from the core with the neutrino luminosity $L_{v}$ can be estimated from a simple relation $L_{v}=\dot{M}_{\mathrm{c}} c^{2}$,

$$
\begin{aligned}
\dot{M}_{\mathrm{c}} & =\frac{L_{v}}{c^{2}} \\
& =6.8 \times 10^{-3}\left(\frac{L_{v}}{10^{11} L_{\odot}}\right) M_{\odot} \mathrm{yr}^{-1},
\end{aligned}
$$

where $c$ is the speed of light.

To estimate the mass-loss rate from the stellar core by the neutrino emission, we numerically followed stellar evolution by using a public stellar evolution code MESAstar version 5527 (Paxton et al. 2011, 2013). We followed the evolution of solar metallicity $(Z=0.02)$ non-rotating stars whose zero-age mainsequence (ZAMS) mass is $25 M_{\odot}$ and $50 M_{\odot}$. We followed the evolution of the stars until the core collapse. The mixing-length parameter is set as $\alpha_{\mathrm{MLT}}=1.6$. We used the MLT ++ module for the convection to follow the evolution up to the core collapse. The Ledoux criterion is adopted for the convection with the dimension-less semiconvection efficiency of $\alpha_{\mathrm{sc}}=1.0$ without overshooting. The wind mass loss from the stellar surface is based on Vink et al. (2001) for the stars with the surface effective temperature above $10^{4} \mathrm{~K}$. The mass-loss rates were

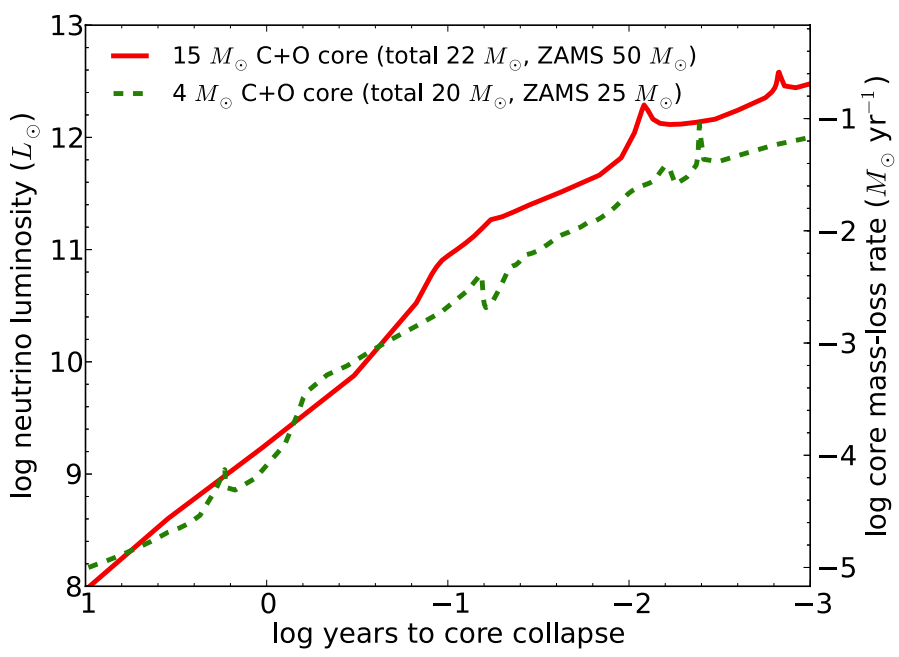

Fig. 1. Neutrino luminosities of massive stars shortly before the core collapse. The righthand side vertical axis shows the corresponding core mass-loss rate, which is estimated with Eq. (2). The mass-loss rate from the stellar surface can be as high as the core mass-loss rate if the star is near the Eddington luminosity.

reduced by 0.8 from the original Vink et al. (2001) prescription (Maeder \& Meynet 2001). For the lower temperatures, we adopted the prescription by de Jager et al. (1988). Neutrino emission in the code was evaluated based on Itoh et al. (1996). At the time of the core collapse, the $25 M_{\odot}$ star has evolved to a $20 M_{\odot}$ red supergiant with a $4 M_{\odot}$ carbon+oxygen core, while the $50 M_{\odot}$ star has become a $22 M_{\odot}$ star with a $15 M_{\odot}$ carbon+oxygen core and a helium-rich layer outside.

Figure 1 presents the neutrino luminosities obtained from the stellar evolution models. Most of neutrinos are thermally emitted and the luminosity increases as the core temperature increases. The obtained neutrino luminosity is consistent with those estimated from other models (e.g., Odrzywolek et al. 2011, 2004). We also show the corresponding mass-loss rate from the stellar core due to the neutrino emission on the righthand axis of Fig. 1. The total mass lost from the core by the neutrino emission within a year before the core collapse is $1.4 \times 10^{-3} M_{\odot}$ and $3.3 \times 10^{-3} M_{\odot}$ in the $25 M_{\odot}$ and $50 M_{\odot}$ models, respectively.

We present the stellar models to estimate the typical neutrino luminosities of massive stars immediately before the explosion. The Eddington factor $\Gamma$ of the $25 M_{\odot}$ and $50 M_{\odot}$ models at the core collapse are 0.0033 and 0.98 , respectively. These stars need to be closer to the Eddington luminosity to enhance mass loss by the central neutrino emission (see Sect. 4). However, the central neutrino emission is not expected to change by the stellar surface luminosity, and the stars whose luminosities are high enough to enhance mass loss should have similar neutrino luminosities to those obtained in this section.

\section{Effect of neutrino mass loss on the stars near the Eddington luminosity}

We now consider a star shortly before the core collapse with mass $M_{\star}$, radius $R_{\star}$, and luminosity $L_{\star}$. The Eddington luminosity $L_{\text {Edd }}$ of the star is expressed as

$L_{\mathrm{Edd}}=\frac{4 \pi G c M_{\star}}{\kappa}$,

where $G$ is the gravitational constant and $\kappa$ the opacity. Here, we consider the stars whose Eddington factor $\Gamma \equiv L_{\star} / L_{\text {Edd }}$ is near 

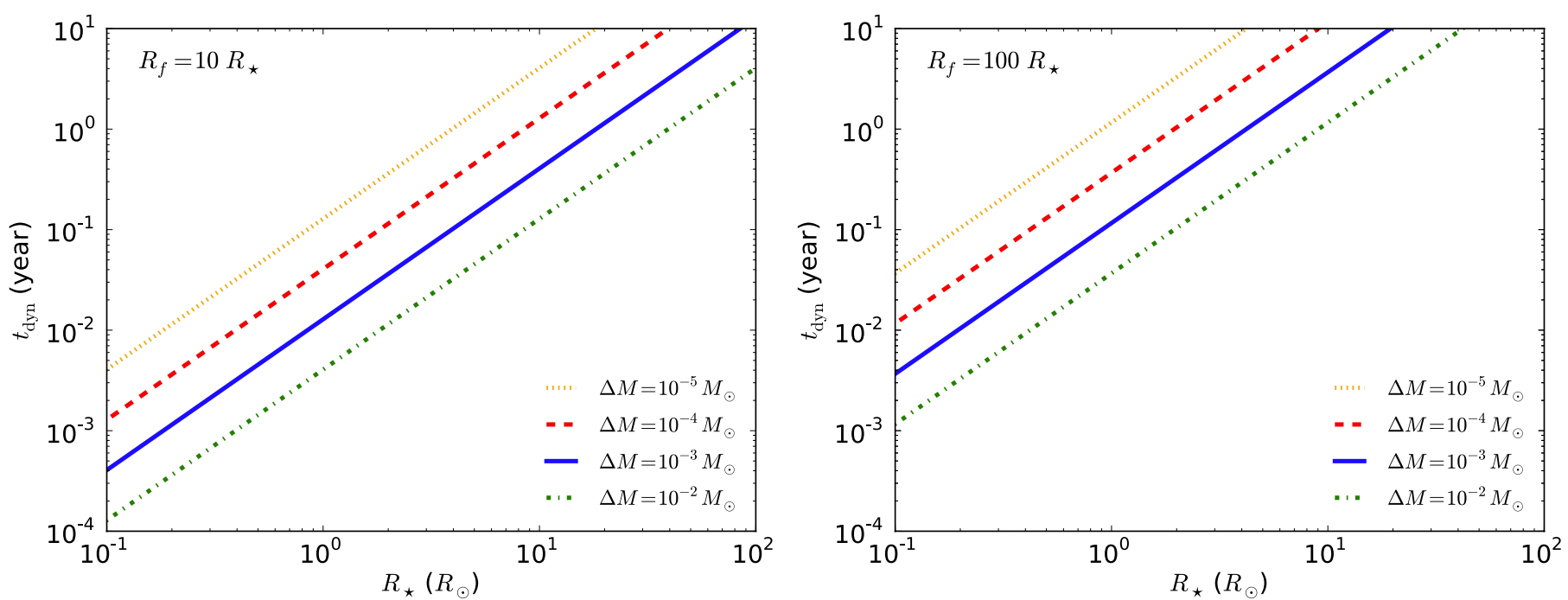

Fig. 2. Dynamical time $t_{\text {dyn }}$ of the material ejected from a stellar surface because of the over-Eddington luminosity. $\Delta M$ is the mass lost from the stellar core. Left panel shows the dynamical time required to reach $10 R_{\star}$ from $R_{\star}$. Right panel shows the dynamical time required to reach $100 R_{\star}$ from $R_{\star}$

unity $(\Gamma \approx 1)$. If a stellar surface is at the Eddington luminosity, the gravitational force and the radiation pressure are balanced. Thus, if the gravitational force is suddenly reduced, the radiation pressure gets higher than the gravitational force, initiating the stellar mass loss. The Eddington luminosity is reduced linearly as neutrinos escape (Eq. (3)).

The resulting mass-loss rate from the stellar surface can be as high as the central mass-loss rate by the neutrino emission because the gravitational energy released is available to push the surface. This means that the star near the Eddington luminosity can have mass-loss rates as high as $10^{-4} M_{\odot} \mathrm{yr}^{-1}$ from about one year before the explosion because of the central neutrino emission (Fig. 1). The line-driven wind mass-loss rates are typically below $10^{-4} M_{\odot} \mathrm{yr}^{-1}$, and the mass loss by the neutrino emission can be dominant from about a year before the core collapse. Overall, the mass-loss rate increases as the star gets closer to the time of the core collapse. The mass-loss rate can exceed $10^{-3} M_{\odot} \mathrm{yr}^{-1}$, which is a typical mass-loss rate for Type IIn SN progenitors from around $\sim 0.1$ years before the core collapse. The following central mass-loss rate strongly depends on the core mass. It can exceed $10^{-2} M_{\odot} \mathrm{yr}^{-1}$ from $\sim 10$ days before the core collapse if the star has a massive core.

Since the high mass-loss rates by the super-Eddington luminosity are achieved within about a year before the core collapse, it is not trivial whether the surface material can actually escape far away from the star within about a year. We assume that $\Delta M$ is reduced from the core by the neutrino emission. As both the gravitational force and the radiative flux are inversely proportional to the square of the distance, the motion of the material leaving the stellar surface is governed by the following equation of motion,

$$
\begin{aligned}
\frac{\mathrm{d}^{2} r}{\mathrm{~d} t^{2}} & =-\frac{G\left(M_{\star}-\Delta M\right)}{r^{2}}+\frac{G M_{\star} \Gamma}{r^{2}}, \\
& =\frac{G M_{\star}}{r^{2}}\left(\frac{\Delta M}{M_{\star}}-1+\Gamma\right), \\
& \approx \frac{G \Delta M}{r^{2}} .
\end{aligned}
$$

$\Gamma \approx 1$ is assumed in deriving Eq. (6). For the moment, we neglect the time dependence of $\Delta M$ for simplicity. By integrating
Eq. (6), we can estimate the dynamical timescale $t_{\text {dyn }}\left(R_{f}\right)$ with which the material ejected from $R_{\star}$ reaches $R_{f}$,

$t_{\mathrm{dyn}}\left(R_{f}\right)=\sqrt{\frac{R_{\star}^{3}}{2 G \Delta M}}\left(\frac{\sin \theta_{f}}{\cos ^{2} \theta_{f}}+\ln \left[\tan \left(\frac{\theta_{f}}{2}+\frac{\pi}{4}\right)\right]\right)$,

where

$\theta_{f}=\arccos \left(\sqrt{\frac{R_{\star}}{R_{f}}}\right) \quad\left(0 \leq \theta_{f}<\frac{\pi}{2}\right)$.

Figure 2 presents the dynamical timescales. The lefthand panel shows $t_{\text {dyn }}\left(10 R_{\star}\right)$ for given $R_{\star}$ and $\Delta M$, and the righthand panel shows $t_{\text {dyn }}\left(100 R_{\star}\right)$. We can see that material ejected from a star with $R_{\star} \sim R_{\odot}$, which is a typical radius of WR stars, can easily reach above $10 R_{\odot}$ within several days and above $100 R_{\odot}$ in $\sim 10$ days. Material ejected from larger stars requires more time to travel. Material ejected from blue supergiants or luminous blue variables (LBVs) $\left(R_{\star} \sim 10 R_{\odot}\right)$ is still able to reach beyond $10 R_{\star}$ within a year. Material ejected from red supergiants with $R_{\star} \sim 100 R_{\odot}$ instead remains near the progenitor at the time of their explosion.

We have neglected the time dependence of $\Delta M$ in deriving $t_{\text {dyn }}$. In reality, $\Delta M$ increases with time. Since the dynamical timescale becomes smaller as $\Delta M$ increases (Fig. 2), the previous arguments obtained by assuming constant $\Delta M$ are presumed not to differ much even if we take the time dependence into account.

Finally, the thermal adjustment timescale of the star,

$$
\begin{aligned}
t_{\mathrm{thm}} & =\frac{G M_{\star}^{2}}{R_{\star} \Gamma L_{\mathrm{Edd}}}, \\
& =4800\left(\frac{M_{\star}}{10 M_{\odot}}\right)\left(\frac{R_{\star}}{R_{\odot}}\right)^{-1}\left(\frac{\kappa}{0.2 \mathrm{~cm}^{2} \mathrm{~g}^{-1}}\right) \Gamma^{-1} \mathrm{yrs}
\end{aligned}
$$

is much larger than the dynamical timescales estimated above and the material pushed by the exceeded radiative force can escape from the system before the adjustment. 


\section{Discussion}

Some progenitors of Type IIn SNe are known to be near the Eddington luminosity (e.g., Gal-Yam \& Leonard 2009; Smith et al. 2011). The high luminosity is often related to LBVs, which are known to experience the enhanced mass loss required to explain the observational properties of Type IIn SNe (e.g., Smith 2006). However, LBVs are not SN progenitors in standard stellar evolution theory (e.g., Langer 2012). Even if LBVs are actually SN progenitors, the unknown mechanism that induces the extreme mass loss observed in LBVs needs to be activated shortly before the core collapse by chance. However, we suggest that, if a SN progenitor is near the Eddington luminosity from several years before the core collapse, the star is not required to be LBVs to enhance the mass loss. The sudden luminosity increase observed in some Type IIn SNe in years to $~ 10$ days before the explosion is naturally expected from the high mass-loss rates of the stellar cores caused by the high neutrino luminosity.

An important characteristic of the mass-loss mechanism presented here is that it can make both hydrogen-rich and hydrogenpoor dense CSM. It induces extreme mass loss shortly before the explosion of a star independently of the surface composition. The stellar luminosity only needs to be close to the Eddington luminosity. There is growing evidence of hydrogen-poor dense CSM around some SN progenitors. Several Type Ib/c SNe show the signatures of the interaction with hydrogen-poor dense CSM (e.g., Pastorello et al. 2007; Gorbikov et al. 2013; Ben-Ami et al. 2013). Some Type Ic superluminous $\mathrm{SNe}$ are also said to be powered by the interaction between dense hydrogen-poor dense CSM and SN ejecta (e.g., Benetti et al. 2013; Chatzopoulos et al. 2013; Moriya \& Maeda 2012; Leloudas et al. 2012; Quimby et al. 2011). However, the mechanisms for making the dense hydrogen-poor CSM suggested so far, such as the pulsational pair instability, can only affect massive stars in a limited range of mass (e.g., Woosley et al. 2007; Chatzopoulos \& Wheeler 2012). Our mechanism for enhancing the mass loss can be activated in a wider range of mass. A WR star just needs to be near the Eddington luminosity shortly before its explosion to have a very high mass-loss rate.

How close should the star be to the Eddington luminosity to activate the mass loss due to the neutrino emission? The Eddington factor $\Gamma$ needs to exceed one when the stellar core mass is decreasing. This means that $\Gamma$ should be larger than $1-\Delta M / M_{\star}$ when the neutrino luminosity becomes high. Assuming $\Delta M \sim 10^{-3} M_{\odot}$ and $M_{\star} \sim 10 M_{\odot}, \Gamma \gtrsim 1-10^{-4}$ needs to be satisfied shortly before the core collapse to enhance the mass loss by the neutrino emission. Note that we are interested in massive stars shortly before the core collapse here. If a massive star is born near the Eddington luminosity, it gets much closer to the Eddington luminosity as it evolves since it loses mass during its evolution by the normal stellar wind. As the Eddington luminosity decreases with mass, the star can get much closer to the Eddington luminosity at the time of the core collapse. Thus, the required proximity to the Eddington luminosity is plausible, especially in the later stage of the star we are interested in.

The mass loss caused by the central neutrino emission may tend to be activated in metal-rich environments. This is because the opacity is expected to be higher in the metal-rich environments. Then the Eddington luminosity can get lower and a star may reach the Eddington luminosity more easily. In addition, the normal wind mass loss that helps a star reach the Eddington luminosity is weakened in metal-poor environments. However, the mass-loss mechanism by the core neutrino emission itself can be activated regardless of the stellar metallicity, and it can enhance the mass loss even in metal-poor environments.

If the star is $\Gamma<1-\Delta M / M_{\star}$, material at the stellar surface does not escape from the star. The reduced gravitational force by the neutrino emission may still affect the stellar structure. The star is presumed to expand because of the reduced binding force. Thus, the stellar radii of SN progenitors can be larger than those expected from stellar evolution theory. However, the reduced core mass is typically $\Delta M \sim 10^{-3} M_{\odot}$ in our models, and the amount of the surface expansion may be small.

\section{Conclusion}

We suggest a new mechanism that can induce high mass-loss rates shortly before the explosion of massive stars. The neutrino luminosities of massive stars increase as they advance the nuclear burning stage. Neutrinos escape freely from the stellar core and take mass out of the core. The core's mass-loss rate by the neutrino emission becomes higher than $10^{-4} M_{\odot} \mathrm{yr}^{-1}$ about a year before the core collapse. The core mass-loss rate becomes higher than $10^{-2} M_{\odot} \mathrm{yr}^{-1} \sim 10$ days before the core collapse (Fig. 1). If a star is close enough to the Eddington luminosity at that time, the stellar luminosity can exceed the Eddington luminosity by the core mass loss because of the reduced gravitational force. Then, the radiative force that was balanced by the gravitational force can push the material at the stellar surface outward and drive strong mass loss. Since the reduced gravitational energy is available to push the surface meterial, the mass-loss rate at the stellar surface can be as high as the core mass-loss rate.

The extreme mass loss by the core neutrino emission can occur even if the star does not have a hydrogen-rich envelope, as long as it is near the Eddington luminosity. Thus, the existence of hydrogen-poor, as well as hydrogen-rich, dense CSM recently observed in some $\mathrm{SNe}$ is naturally expected from this mechanism. Even if a star is not close enough to the Eddington luminosity to enhance the mass loss by the neutrino emission shortly before the core collapse, it can still expand because of the reduced gravitational force. This indicates that some stars may have larger radii at the time of the explosion than those expected from the stellar evolution theory.

Acknowledgements. I would like to thank the referee for the comments that improved this work significantly. The author is supported by the Japan Society for the Promotion of Science Research Fellowship for Young Scientists (23.5929). Numerical computations were carried out on computers at Center for Computational Astrophysics, National Astronomical Observatory of Japan.

\section{References}

Balberg, S., \& Loeb, A. 2011, MNRAS, 414, 1715

Ben-Ami, S., Gal-Yam, A., Mazzali, P. A., et al. 2013 [arXiv:1309.6496]

Benetti, S., Nicholl, M., Cappellaro, E., et al. 2013, MNRAS, in press [arXiv: 1310.1311]

Bersten, M. C., Tanaka, M., Tominaga, N., Benvenuto, O. G., \& Nomoto, K. 2013, ApJ, 767, 143

Chatzopoulos, E., \& Wheeler, J. C. 2012, ApJ, 760, 154

Chatzopoulos, E., Wheeler, J. C., Vinko, J., Horvath, Z. L., \& Nagy, A. 2013, ApJ, 773, 76

Couch, S. M., Pooley, D., Wheeler, J. C., \& Milosavljević, M. 2011, ApJ, 727, 104

de Jager, C., Nieuwenhuijzen, H., \& van der Hucht, K. A. 1988, A\&AS, 72, 259

Dessart, L., Hillier, D. J., Livne, E., et al. 2011, MNRAS, 414, 2985

Fraser, M., Inserra, C., Jerkstrand, A., et al. 2013a, MNRAS, 433, 1312

Fraser, M., Magee, M., Kotak, R., et al. 2013b, ApJ, 779, L8

Gal-Yam, A., \& Leonard, D. C. 2009, Nature, 458, 865

Gorbikov, E., Gal-Yam, A., Ofek, E. O., et al. 2013, MNRAS, submitted [arXiv: 1312.0012] 
T. J. Moriya: Mass loss of SN progenitors by core neutrino emission

Itoh, N., Hayashi, H., Nishikawa, A., \& Kohyama, Y. 1996, ApJS, 102, 411 Kiewe, M., Gal-Yam, A., Arcavi, I., et al. 2012, ApJ, 744, 10

Kleiser, I. K. W., \& Kasen, D. 2013, MNRAS, 2894

Langer, N. 2012, ARA\&A, 50, 107

Leloudas, G., Chatzopoulos, E., Dilday, B., et al. 2012, A\&A, 541, A129

Levan, A. J., Tanvir, N. R., Starling, R. L. C., et al. 2014, ApJ, 781, 13

Lovegrove, E., \& Woosley, S. E. 2013, ApJ, 769, 109

Maeder, A., \& Meynet, G. 2001, A\&A, 373, 555

Margutti, R., Milisavljevic, D., Soderberg, A. M., et al. 2014, ApJ, 780, 21

Mauerhan, J. C., Smith, N., Filippenko, A. V., et al. 2013, MNRAS, 430, 1801

Moriya, T. J., \& Maeda, K. 2012, ApJ, 756, L22

Moriya, T. J., Maeda, K., Taddia, F., et al. 2013, MNRAS, 435, 1520

Nadezhin, D. K. 1980, Ap\&SS, 69, 115

Odrzywolek, A., Misiaszek, M., \& Kutschera, M. 2004, Astropart. Phys., 21, 303

Odrzywolek, A., Misiaszek, M., \& Kutschera, M. 2011, Nucl. Phys. B Proc. Suppl., 221, 380
Ofek, E. O., Sullivan, M., Cenko, S. B., et al. 2013, Nature, 494, 65 Pastorello, A., Smartt, S. J., Mattila, S., et al. 2007, Nature, 447, 829 Pastorello, A., Cappellaro, E., Inserra, C., et al. 2013, ApJ, 767, 1

Paxton, B., Bildsten, L., Dotter, A., et al. 2011, ApJS, 192, 3

Paxton, B., Cantiello, M., Arras, P., et al. 2013, ApJS, 208, 4

Prieto, J. L., Brimacombe, J., Drake, A. J., \& Howerton, S. 2013, ApJ, 763, L27

Quataert, E., \& Shiode, J. 2012, MNRAS, 423, L92

Quimby, R. M., Kulkarni, S. R., Kasliwal, M. M., et al. 2011, Nature, 474, 487

Shiode, J. H., \& Quataert, E. 2014, ApJ, 780, 96

Smith, N. 2006, ApJ, 644, 1151

Smith, N., Li, W., Silverman, J. M., Ganeshalingam, M., \& Filippenko, A. V. 2011, MNRAS, 415, 773

Smith, N., Mauerhan, J., \& Prieto, J. 2014, MNRAS, 438, 1191

Taddia, F., Stritzinger, M. D., Sollerman, J., et al. 2013, A\&A, 555, A10

Tauris, T. M., Langer, N., Moriya, T. J., et al. 2013, ApJ, 778, L23

Vink, J. S., de Koter, A., \& Lamers, H. J. G. L. M. 2001, A\&A, 369, 574

Woosley, S. E., Blinnikov, S., \& Heger, A. 2007, Nature, 450, 390 